\title{
Modeling of particles migration in porous media: application to soil suffusion
}

\author{
C. Ahmed \\ University of Sciences and Technology of Oran "Mohammed Boudiaf", Algeria \\ B. Ahmed \\ LOMC UMR 6294 CNRS - Université du Havre, 53 rue de Prony 76600 Le Havre, France
}

\begin{abstract}
The suffusion phenomenon occurs when fine soil particles are detached by seepage flow and transported away from the matrix. This process is one of the main causes of failure of hydraulic structures and road embankments. This study aimed to build a numerical model for simulating the particles suffusion within a porous medium. This model combines a flow law and an erosion equation related to the evolution of soil porosity. In addition the dispersion and the deposition kinetics processes of eroded particles were combined with detachability process. The equations describe the evolution of the instantaneous concentration of the fluidized solid, and the variation of eroded mass. Sensitivity analysis allows highlighting the influence of the different parameters on suffusion, particularly that deposition kinetics starts acting only below a threshold of hydraulic gradient and beyond a given sample length. The adjustment results indicate that the suffusion process is strongly related to hydraulic conditions, physical soil characteristics and pore water chemicals. The comparison of numerical results with experimental data from laboratory tests provides a quite good agreement.
\end{abstract}

\section{INTRODUCTION}

Suffusion can occasionally occur in internally unstable soils that are subject to seepage flow. Suffusion (erosion occurring within the volume medium) of a soil is the process whereby finer particles migrate within the void network of its coarser particles, as a result of seepage flow. Onset of internal erosion is governed by a combination of geometric and hydromechanical conditions. Migration of fine particles to an unprotected exit, from which the eroded material may escape, by washing out, could cause erosion or piping failure. The internal erosion is one of the main causes of failure of hydraulic structures. From the reviewed literature the suffusion is a dangerous process that threatens the stability of hydraulic structures, since about $50 \%$ of internal erosion incidents are due to suffusion. This process involves the detachment and transport of fine particles through soil matrix, leading to solid volume reduction. However, even particles are released from soil they could be trapped within pore structure, reducing the suffusion kinetics. Then, transport and attachment parameters must be taken into account in suffusion models.

Numerical modelling is therefore an essential tool of the forecast study of suffusion process. In this context, several mathematical models were presented. Govindaraju (1995) represents the erosion evolution by three distinct phases and each one is gov- erned by its specific ordinary differential equation as erosion law. The model is reduced to an equation describing the temporal evolution of the product of porosity and concentration, neglecting the spatial evolution of the dispersion of the particles in the porous medium and also the deposition process. Based on this model, Seghir A. et al. (2014) used a simplified form of Govindraju model in order to derive analytical solution. The results compared with experimental data obtained from suffusion in sand-fines mixtures show a quite good agreement. Roy \& Dzombak (1996) presented a model devoted to simulate colloid release and transport processes in porous media, based on advection-dispersion equation with colloid deposition and release terms. But, in their model, the authors assume that the porosity does not change as result of colloid deposition and release. Such model is not suitable to large erosion experienced by hydraulic works. Vardoulakis et al. (1996) and Papamichos \& Vardoulakis (2005) presented a macroscopic model for incompressible fluid flow which was used to investigate the sand production problem in oil wells. However, this model seems not be applied successfully to the suffusion process because it involves continuous release of particles until the entire solid matrix is eroded. Sterpi (2003) formulated a mathematical model based on empirical erosion law where the eroded fine fraction is related to the initial fine fraction and the hydraulic gradient. This erosion law is an increasing function 
over time that can reach the entire initial fine fraction in the soil when time increases infinitely, and according to the author, this is valid under even low hydraulic gradient value. Gravelle et al. (2011) developed a model based on the advection-dispersion equation where deposition process is neglected. In this model, because one term release rate is not enough to obtain a good correlation with experimental data, a second order term of release rate was added. Bonelli \& Marot (2011) presented a model where suffusion occurs at pore scale as an interfacial process. The model depends on the threshold hydraulic gradient which is determined empirically. Water in the soil contains soluble salts whose concentration determines whether the soil is "normal" or "salt-affected". Chemical conditions can be a concern when dams and soils pore water become more saline. It was reported from literature that soil suffusion rate is strongly affected by the ionic strength of pore solution (Khilar and Fogler 1984; Roy \& Dzombak 1996; Blume et al. 2005).

The reported models display mainly processes of particles detachment, involving many parameters to describe the suffusion process. But particles detached from soil matrix are also submitted to the main transport processes occurring in porous media like dispersion and deposition which are affected by the porous medium grading and also by flowing fluid chemicals. So, particles removed from matrix are not all washed out from the soil and may be retained within the pores, leading to likely clogging. In order to take into account the main processes of particles release and transport within the porous medium (detachment and retention in soil matrix), a mathematical model devoted to describe the suffusion process in a soil submitted to hydraulic load is presented. This model is based on advection-dispersiondeposition equation coupled to an erosion law describing the evolution of soil porosity. Because the deposition kinetics can be related to pore water salinity, another skill investigated in this study is fluid salinity effect on fines erodibility.

\section{MATHEMATICAL MODEL OF SUFFUSION}

The model describes the suffusion and transport processes (including particle deposition) in a porous medium submitted to one dimensional flow, which is suitable for tracer tests in column. Assessed equations use macroscopic laws relating pressure, water velocity and external forces at the scale of representative elementary volume, and coupled Darcy's law to an erosion law which depends on the soil porosity variation.

We assume that the initial sample consists of twophases: solid $s$ and fluid $f$. At the initiation of erosion a third phase raises $f_{s}$ (fluidized fine particles) with- in the medium volume, This phase is characterized by the volume concentration $C$ of eroded particles.

It is usual to assume that the solid densities of sand and silt, denoted $\rho_{s}$, as being identical and close to $2600 \mathrm{~kg} / \mathrm{m}^{3}$.

\subsection{Balance equations}

As the medium was assumed to be consisting of three phases, the mass conservation equation is applied for each phase. The suffusion occurs by the solid mass lost and so the principle of solid mass conservation can be written as the balance between solid mass variation and source term (lost solid matter):

$\frac{\partial\left[(1-\emptyset) \rho_{s}\right]}{\partial t}=-j$

Where:

$j$ : Mass flow rate from erosion, per unit volume $\left(\mathrm{kg} / \mathrm{m}^{3} / \mathrm{s}\right)$

$\emptyset$ : Instantaneous porosity of soil

The conservation law of the fluidizable solid mass $\rho_{s} \varnothing C$ in pore volume involves the displacement velocity $\mathrm{u}_{f s}$ of fluidized solid (eroded fine particles) which is submitted to dispersion and deposition processes:

$$
\begin{aligned}
\frac{\partial\left(\varnothing \rho_{s} C\right)}{\partial t}+\frac{\partial\left[\left(\varnothing \rho_{s} C\right) u_{f s}\right]}{\partial x} & \\
& =j+D \frac{\partial^{2}\left(\varnothing \rho_{s} C\right)}{\partial x^{2}}-k_{d}\left(\varnothing \rho_{s} C\right)
\end{aligned}
$$

Where $C$ is the volume concentration of eroded particles (volume fraction), $D$ is the dispersion coefficient $\left(\mathrm{m}^{2} / \mathrm{s}\right)$ and $K_{d}$ is the deposition kinetics coefficient $\left(\mathrm{s}^{-1}\right)$.

As usually assumed, the dispersion coefficient $D$ behaves linearly with pore velocity $u=\frac{q}{\varnothing}$ through the (longitudinal) dispersivity coefficient $\alpha$, expressed by: $D=\alpha$.u.

Also, the conservation of fluid mass $\rho_{f}(1-C) \emptyset$ provides the following equation where the source term is null because there is no input or loss of fluid mass within the system:

$\frac{\partial\left(\rho_{f}(1-C) \emptyset\right)}{\partial t}+\frac{\partial\left[\left(\rho_{f}(1-C) \emptyset\right) u\right]}{\partial x}=0$

Since suffusion involves transport of fine particles (fluidized phase), we can assume that their velocity is equal to that of fluid phase $\left(u=u_{s f}\right)$ and using the relation between Darcy velocity and pore velocity $(q=\varnothing u)$, Eqs. (1) to (3) led to: 


$$
\left\{\begin{array}{c}
\frac{\partial \emptyset}{\partial t}=\frac{j}{\rho_{s}} \\
\frac{\partial\lfloor(C-1) \emptyset\rfloor}{\partial t}+\frac{\partial(q C)}{\partial x}=D \frac{\partial^{2}(\varnothing C)}{\partial x^{2}}-k_{d}(\varnothing C)
\end{array}\right.
$$

Assuming that the flow is incompressible:

$\frac{\partial q}{\partial x}=0$

Then the previous system of equations (4) is equivalent to the following one:

$$
\left\{\begin{array}{l}
\frac{\partial \emptyset}{\partial t}=\frac{j}{\rho_{s}} \\
\frac{\partial((C-1) \emptyset)}{\partial t}+q \frac{\partial C}{\partial x}=D \emptyset \frac{\partial^{2} C}{\partial x^{2}}-k_{d} . \emptyset C \\
\frac{\partial q}{\partial x}=0
\end{array}\right.
$$

The second equation of the system (6) describes the advective-dispersive and deposition processes occurring during suffusion.

\subsection{Behaviour laws}

\subsubsection{Flow law}

Since the fluid velocity within the soil remains very low (laminar regime) Darcy's law can be applied:

$q=-k \cdot \operatorname{grad} H$

Where $\mathrm{H}$ is the hydraulic head and $k$ the hydraulic permeability related to intrinsic permeability through the following equation:

$k=K \cdot \gamma_{w} / \mu$

where $K$ is the intrinsic permeability $\left(m^{2}\right), \mu$ the dynamic viscosity of water $\left(K g \cdot \mathrm{m}^{-1} \cdot \mathrm{s}^{-1}\right)$ and $\gamma_{w}$ the specific weight of water $\left(\mathrm{N} . \mathrm{m}^{-3}\right)$.

The intrinsic permeability can be related to porosity and the formulation suggested by Kozeny-Carman (Carman 1937; Koponen et al. 1997; Barnichon 2000; Chapuis and Aubertin 2003; Trani and Indraratna 2010; Berg 2014) is used:

$K=C_{k} \frac{\emptyset^{3}}{(1-\emptyset)^{2}}$

Where $C_{k}$ is a constant obtained by Eq. 9 for the initial conditions $\left(K_{0}\right.$ and $\left.\emptyset_{0}\right)$.

Then, Eq. 9 can be rewritten as follows:
$K=K_{0} \frac{\left(1-\emptyset_{0}\right)^{2}}{\emptyset_{0}^{3}} \frac{\emptyset^{3}}{(1-\emptyset)^{2}}$

So, Darcy equation (Eq. 7) can be expressed through initial values of soil parameters:

$q=-K_{0} \frac{\gamma_{w}}{\mu} \frac{\left(1-\emptyset_{0}\right)^{2}}{\emptyset_{0}^{3}} \frac{\emptyset^{3}}{(1-\emptyset)^{2}} \operatorname{grad} H$

Furthermore:

$K_{0}=k_{0} \frac{\mu_{0}}{\gamma_{w}}$

With:

$k_{0}$ : Initial hydraulic Permeability $(\mathrm{m} / \mathrm{s})$

$\mu_{0}$ : Initial dynamic viscosity of water $\left(\mathrm{Kg}_{\mathrm{m}} \mathrm{m}^{-1} \cdot \mathrm{s}^{-1}\right)$

So:

$q=-k_{0} \frac{\mu_{0}}{\mu} \frac{\left(1-\emptyset_{0}\right)^{2}}{\emptyset_{0}^{3}} \frac{\emptyset^{3}}{(1-\emptyset)^{2}} \operatorname{grad} H$

\subsubsection{Erosion law}

Since the eroded mass is produced by the fine mass fraction per unit volume $\rho_{s} R_{\text {fine }}$, it is assessed here that the erodible fine fraction is a function depending on the evolution of the porosity with respect to its initial value:

$R_{\text {fine }}(t)=f\left(\phi_{0}, \phi(t)\right)=C_{m e} \phi_{0}-\phi(t)$

The erosion flux becomes:

$j=\lambda \rho_{s} R_{\text {fine }} q=\lambda \rho_{s}\left(C_{m e} \phi_{0}-\phi\right)|q|$

With:

$\lambda$ : coefficient of erosion $\left(\mathrm{m}^{-1}\right)$;

$R_{\text {fine }}:$ volume fraction of the erodible fine fraction;

$\emptyset_{0}$ : initial porosity.

$C_{m e}$ : maximum erodibility coefficient.

Thus, the following system of equations is obtained:

$\left\{\begin{array}{c}\frac{\partial \emptyset}{\partial t}=\lambda\left(C_{m e} \phi_{0}-\phi\right) q \\ \frac{\partial((C-1) \emptyset)}{\partial t}+q \frac{\partial C}{\partial x}=D \emptyset \frac{\partial^{2} C}{\partial x^{2}}-k_{d} \emptyset C \\ q=-k_{0} \frac{\mu_{0}}{\mu} \frac{\left(1-\emptyset_{0}\right)^{2}}{\emptyset_{0}^{3}} \frac{\emptyset^{3}}{(1-\emptyset)^{2}} \operatorname{gradH}\end{array}\right.$ 


\subsubsection{Rheological behaviour law}

During erosion the viscosity of the interstitial fluid is evolving according to the increase of pore fluid concentration subsequently to particles detachment. Einstein's formula (1906, modified on 1911) is used to assess the evolution of the viscosity for dilute suspension $(C \leq 0.03)$ :

$\mu=\mu_{0}(1+2,5 C)$

Thus, the equations system (16) becomes:

$$
\left\{\begin{array}{c}
\frac{\partial \emptyset}{\partial t}=\lambda\left(C_{m e} \phi_{0}-\phi\right) q \\
\frac{\partial((C-1) \emptyset)}{\partial t}+q \frac{\partial C}{\partial x}=D \frac{\partial^{2}(\emptyset . C)}{\partial x^{2}}-k_{d}(\varnothing C) \\
q=-\frac{k_{0}}{1+2,5 C} \frac{\left(1-\emptyset_{0}\right)^{2}}{\emptyset_{0}^{3}} \frac{\emptyset^{3}}{(1-\emptyset)^{2}} \operatorname{gradH}
\end{array}\right.
$$

It is known that suffusion and filtration are two coupled actions within the soil submitted to seepage. The second equation of above system (18) represents advective-dispersive-deposition processes occurring during suffusion. In what follows, we study the case of constant hydraulic gradient which is the most representative case of the real conditions of an earth dam.

\subsection{Numerical solution}

From the mass conservation and behaviour laws, we obtained a system of differential equations with three unknowns (porosity $\varnothing$, volume concentration of particles in the fluid $C$ and Darcy velocity $q$ ). This model is solved numerically by $1 \mathrm{D}$ upwind finite difference method. The sample is discredited into a set of elementary layers referenced by the index $j$ and the instant by the exponent $i$. Explicit time scheme is used to solve the previous equations. Two appropriate initial conditions are used:

$\phi(0, x)=\phi_{0}, C(0, x)=C_{0}=0$

The boundary conditions adopted are: (i) in upstream of the sample, no fluidized solid in the injected fluid (clear water): $C(t, 0)=0$; (ii) the solid concentration in the fluid at the downstream (outlet) of the sample is equal to the concentration of the last layer:

$$
\frac{\partial C(t, L)}{\partial x}=0
$$

In the following and in order to facilitate comparison with experimental results, the concentration is determined as a function of the mass concentration:

$C_{M}\left[\frac{g}{l}\right]=C \rho_{s}$

\section{RESULTS AND COMMENTS}

\subsection{Sensitivity analysis:}

The model involves many parameters which can affect differentially the computed results. So, the influence of the variation of each parameter is addressed in this section.

The aim of these tests is to determine the sensitivity of the mathematical model toward each parameter, mainly the erosion coefficient, maximum erodibility coefficient and deposition kinetics, which also depend on the hydraulic gradient and the initial fine fraction.

In order to achieve the sensibility analysis, the time-mass concentration variation, provided through the numerical resolution of Eq. (18), and the eroded cumulative mass deduced as: $m=\rho_{s} \int_{0}^{t} C . Q . d t$ or relative cumulative mass $\mathrm{m} / \mathrm{m}_{0}$, are used to assess the influence of model parameters.

\subsubsection{Effect of erosion coefficient}

In the presented model, the main parameter governing detachment of fine particles is the erosion coefficient $\lambda$. The effect of its variation on the model's results is assessed through the temporal evolution of the concentration and the relative cumulative eroded mass.
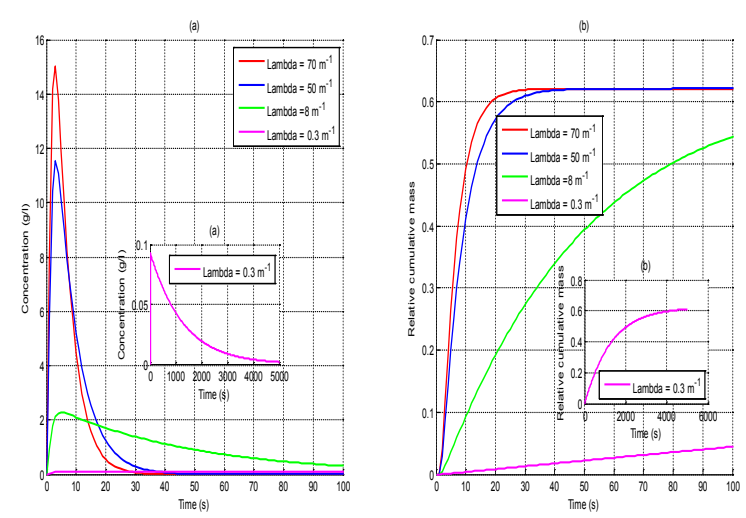

Fig. 1 Temporal evolution of concentration and eroded mass fraction (at the outlet: $\mathrm{x}=\mathrm{L}$ ) for different values of $\lambda$ $(\operatorname{gradH}=6.2, \mathrm{Cme}=1.0176)$

Figure 1 shows that the evolution of suffusion is proportional to the erosion coefficient. Numerical results show that the erosion coefficient affects the erosion rate, mainly within short time. However, we note no significant influence of such parameter on 
final cumulative eroded mass, which is constant for any value of $\lambda$ (Fig. 1(b)). It is clear that the model is sensitive to very low values of $\lambda$ (ie. $\lambda=0.3 \mathrm{~m}^{-1}$ ). For the time-concentration variation (Fig. 1 (a)), it is obtained that the peak of the concentration increases when erosion coefficient increases. However, when erosion coefficient decreases the curve $C(\mathrm{t})$ is flattened and tends to a residual value of suffusion. This indicates that erosion rate is related to erosion coefficient $\lambda$. So, the particles contained in the medium are washed out as rapidly as erosion coefficient value increases.

\subsubsection{Effect of the maximal erodibility coefficient}

The maximal erodibility coefficient $C_{m e}$ expresses the ratio between the maximal soil porosity (which can be reached at the end of erosion) and the initial porosity. Its variation indicates how long a specimen can loss fine particles. Fig. 2 shows the variation of eroded mass with increasing value of $C_{m e}$. It is clear that for the same initial fines fraction $f_{0}$ (same initial porosity), the increase of $C_{m e}$ (increase of $\phi_{\max }$ ) is proportional to the increase of the maximum erodible mass fraction $f_{\max }$ according to the relation:

$f_{\max }=7.467\left(C_{m e}-1\right)$

\subsubsection{Combined effects of deposit coefficient, sam- ple length and hydraulic gradient}

By varying the deposition kinetics coefficient, figure 3 shows the combined effects of variation of deposition coefficient and sample length on the timeconcentration and the eroded mass variation. However, for short length a double value of deposit coefficient (from $K_{d}=0.05 s^{-1}$ to $K_{d}=0.1 s^{-1}$ ) do not produces any increase of eroded mass. In opposite, for a long sample, the influence is very clear. The effect of deposition rate coefficient on suffusion starts arising from a sample length close to $6 \mathrm{~cm}$.

Figure 4 shows the combined effects of variation of deposit kinetics coefficient and hydraulic gradient on suffusion process. It is found that the influence of the variation of the deposition rate depends on the value of the hydraulic gradient. For high hydraulic gradient values $(\mathrm{gradH}=10.7)$, this influence is weak, while under low hydraulic gradient (gradH $=$ 1.07) the influence of deposition kinetics coefficient is strong.

We can conclude that wider is the hydraulic work (lower is the hydraulic gradient), lower is the eroded mass, indicating high deposition of fine particles within the soil matrix.
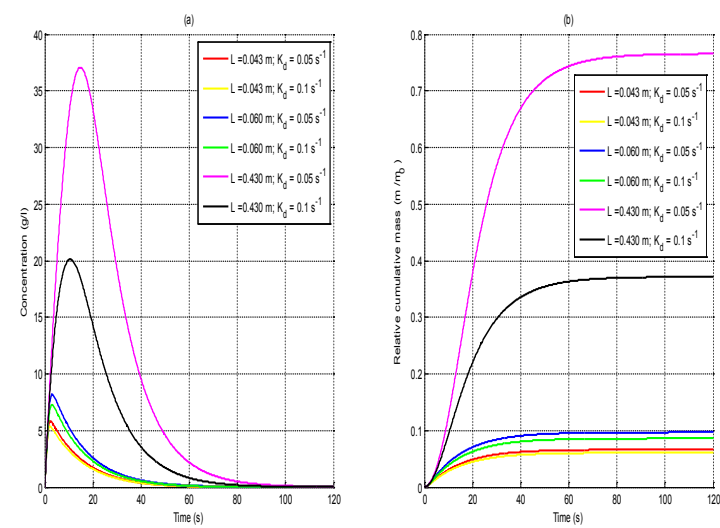

Fig. 3: Combined effect of length and $\mathrm{Kd}$ on concentration and eroded mass fraction (at the outlet: $\mathrm{x}=\mathrm{L})(\operatorname{gradH}=10.72, \lambda=8 \mathrm{~m}$ $1, \mathrm{Cme}=1.027$ )
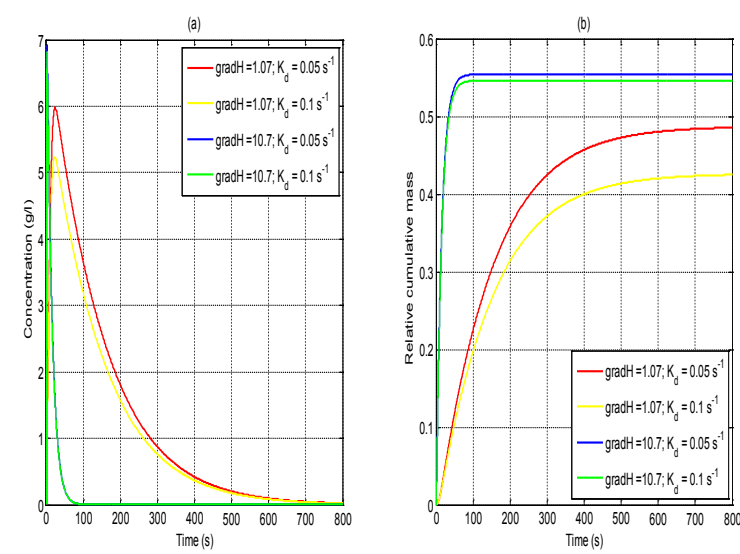

Fig. 4: Combined effect of gradH and $\mathrm{Kd}$ on concentration and eroded mass fraction (at the outlet: $\mathrm{x}=\mathrm{L})(\mathrm{L}=4.3 \mathrm{~cm}, \lambda=8 \mathrm{~m}-1$, $\mathrm{Cme}=1.027$ )

\subsubsection{Coupled effects of dispersion coefficient and sample length}

By varying the dispersivity coefficient value in the model, it is obtained that the production of eroded mass fraction is inversely proportional to the increase of the dispersion (Fig. 5 and 6). So, the influence of dispersivity rises only from value close to $0.1 \mathrm{~cm}$, even if sample length is significantly large. So, the model seems to be not sensitive to the dispersion, since dispersivity value ten times higher decreases final eroded mass only within a range lower than $25 \%$ even for short sample. The amplitude of cumulative eroded mass depends on the variation of other parameters: it is less important in samples of short length, while it is more important for samples of the same length but subject to stronger gradients. For the same hydraulic gradient, the eroded mass fraction is more important for short lengths.

In the process of mass transport through porous media, advection and dispersion are in competition. So, if decreasing hydraulic gradient, the effect of dispersion on eroded mass is reduced. This result is in accordance with reported ones (Benamar et al. 2007; 
Ahfir et al. 2009) regarding the increase of dispersion with increasing pore velocity. The relative influence of advection as regards to dispersion is quantified by hydrodynamic Péclet number, which is defined by:

$P e=\frac{u . L}{D}=\frac{L}{\alpha}$
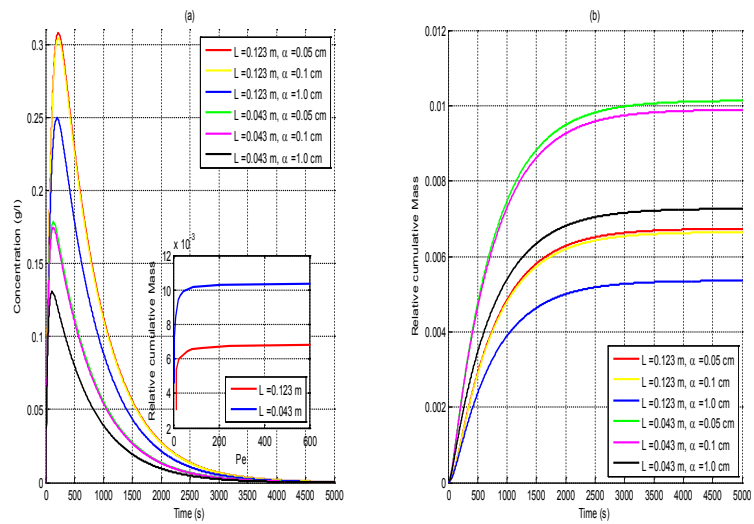

Fig. 5: Combined effect of length and $\alpha$ on suffusion process (at the outlet: $\mathrm{x}=\mathrm{L})(\operatorname{gradH}=4.8)$
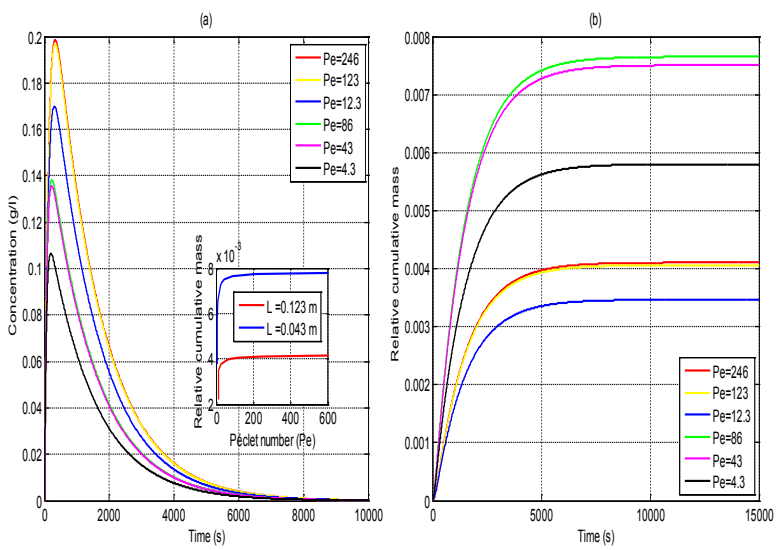

Fig. 6: Combined effect of length and $\alpha$ on suffusion process (at the outlet: $\mathrm{x}=\mathrm{L})(\operatorname{gradH}=2.4)$

As regards to increasing Péclet number, cumulative eroded mass quickly reached an asymptotic value. This evolution indicates that Péclet number has a significant influence on the suffusion process only within the range of its low values (Figs. 5 and 6). By increasing the sample length by three times, the ultimate eroded mass fraction is reduced by about two times. This means that in embankment dams enlarging hydraulic work width may reduce eroded mass fraction (if any). This result is in accordance with the inverse effect of initial fine content (proportional to sample length) on cumulative eroded mass (Seghir A. et al. 2014).

\subsection{Adjustment of experimental results}

\subsubsection{Materials and experimental procedure}

The experimental apparatus consists of a reservoir filled with water which provides a hydraulic load applied to a column containing the soil sample. The outlet of the column is directed to a turbidity meter whose data were recorded and stored by a computer. A flowmeter connected to the column outlet allows measuring the effluent discharge. Differential pressure between upstream and downstream of the soil sample was measured using two piezometers, providing the mean hydraulic gradient along the specimen. Several columns of different sizes were used (table 1), a glass beads layer was placed upstream the sample and a mesh screen $(100 \mu \mathrm{m}$ opening size) was positioned downstream the column. The suffusion tests were performed on a mixture of coarse sand, whose particle size ranges between 0.315 and $1.60 \mathrm{~mm}$, and silt whose particle size ranges mainly between $1 \mu \mathrm{m}$ and $80 \mu \mathrm{m}$.

Table 1 shows the different characteristics of the reconstituted samples. The fines content in mixtures was varied from $2 \%$ to $8 \%$ by weight. The mixtures were prepared by mixing sand and silt with $3 \%$ of water content and left at rest 24 hours before filling the column using double-compaction at the target dry density close to $1800 \mathrm{~kg} / \mathrm{m} 3$. In order to not disturb the soil before starting the test, a water saturation was achieved under a low hydraulic head $(\mathrm{H}=2$ $\mathrm{cm}$ ). The suffusion test involves the initiation of water flow through the specimen under a fixed hydraulic gradient (Fig. 7). Table 1 presents the main characteristics of the materials and the hydraulic gradient under which the sample was tested.

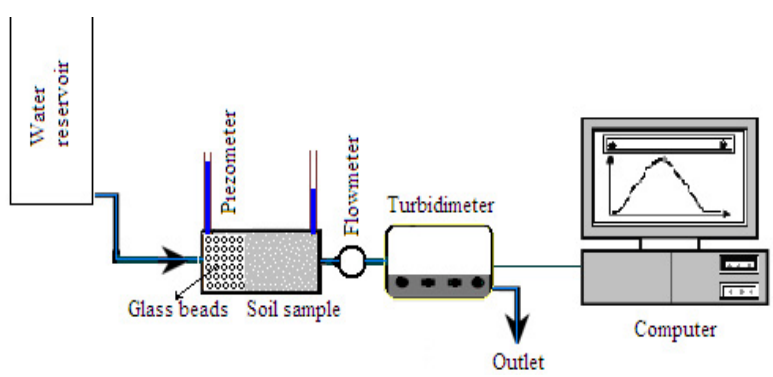

Fig. 7 Experimental setup 
Table 1: Sample characteristics and test conditions

\begin{tabular}{|c|c|c|c|c|c|c|c|c|}
\hline \multirow{2}{*}{ 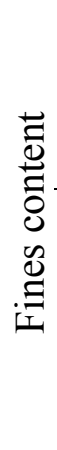 } & din & isions & \multirow{2}{*}{ 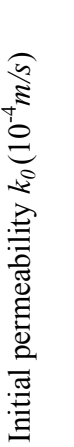 } & \multirow{2}{*}{ 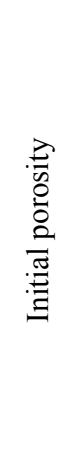 } & \multirow{2}{*}{ 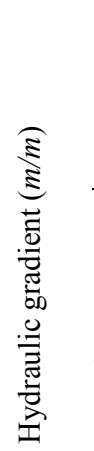 } & \multicolumn{3}{|c|}{$\begin{array}{l}\text { Numerical } \\
\text { parameters }\end{array}$} \\
\hline & 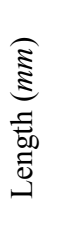 & 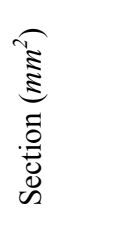 & & & & $\underset{\Sigma}{\Sigma}$ & $\tilde{U}^{\check{E}}$ & 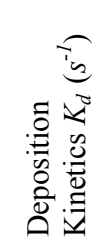 \\
\hline$\overline{8 \%}$ & 35 & 803.75 & 11.5 & 0.32 & 11.7 & 3.7 & 1.04 & 10.010 \\
\hline $6 \%$ & 43 & 803.75 & 7.7 & 0.32 & 10.7 & 76.7 & 1.03 & 50.010 \\
\hline $2 \%$ & 22 & 803.75 & 4.1 & 0.36 & 6.2 & 19 & 1.02 & 00.010 \\
\hline $5 \%$ & 123 & 1256.6 & 0.3 & 0.35 & 2.4 & 8.5 & 1.00 & 10.007 \\
\hline $5 \%$ & 123 & 1256.6 & 0.3 & 0.35 & 2.4 & 4.0 & 1.00 & 20.009 \\
\hline $5 \%$ & 123 & 1256.6 & 0.3 & 0.35 & 2.4 & 1.0 & 1.00 & 60.010 \\
\hline
\end{tabular}

\subsubsection{Model validation:}

In order to validate the model, experimental data obtained from previous tests were faced to the numerical results. The adjustment was performed through the time-mass concentration and the deduced cumulative eroded mass evolution at the outlet of the sample. Fig. 8 shows the adjustment between experimental data and numerical results obtained for three different initial fines content under different hydraulic gradients. Time-concentration evolution (figure $8(\mathrm{a}))$ shows rapid increase of the concentration of the effluent reaching a peak whose magnitude decreases with increasing initial fines content even if hydraulic gradient also increases. These results show that the effect of fines content is predominant than hydraulic gradient in suffusion process. Numerical results provide a good agreement with experimental curves even within the residual value of concentration over the test time. This basic model therefore provides representative curves of the phenomenon of suffusion, recorded experimentally.
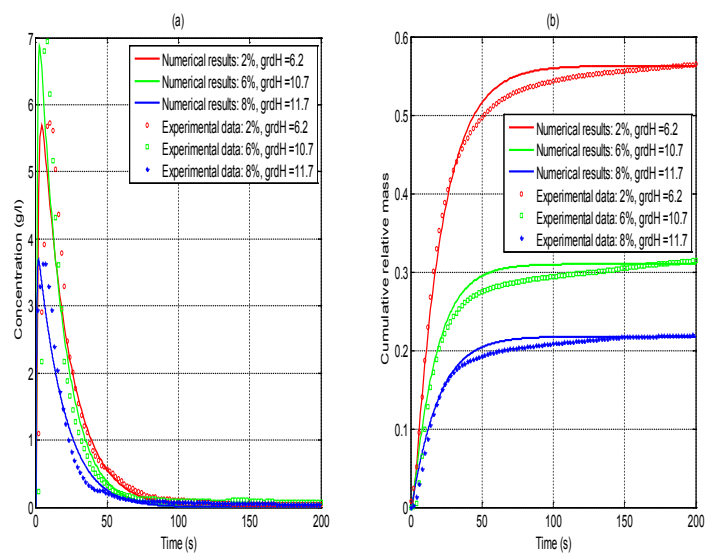

Fig. 8: effect of initial fine fraction on suffusion process (at the outlet: $\mathrm{x}=\mathrm{L})($ Silt: $2 \%, 6 \%$ and $8 \%$ )

If addressing the effect of hydraulic gradient on the eroded fraction of fines, the comparison of the results obtained with several fines contents $(2 \%, 6 \%$ and $8 \%$ ) within samples subject to different hydraulic gradients, showed the increase of eroded mass fraction with decreasing initial fines content, in spite of the decrease of hydraulic gradient. Independently of hydraulic conditions, lower is the initial fines content in the soil, more important is the eroded fraction of fine particles, as reported by Seghir A. et al. 2014. It was also reported (Wan \& Fell (2004)) that in engineering dam the embankments designed with at least $20 \%$ of fines are safe against suffusion. The geotechnical explanation for the effect of fines content can be supported by the soil cohesion (shear strength) involved by higher fraction of fines which lead to make particles attached together and to the matrix. The sensitivity of such parameter (initial fines content) shows that its slight variation causes a large variation in the ultimate eroded mass and so more vulnerability to suffusion. It is then often concluded that more is the initial fines content less the soil is erodible.

\section{SUMMARY AND CONCLUSION}

A simplified model of the suffusion phenomenon, based on a macroscopic approach is presented. Flow rate is assumed to be governed by Darcy's law and the changes in permeability were related to be governed by Kozeny-Carman equation. This model is solved numerically by forward finite difference method 1D. The influence of the variation of the physical parameters (hydraulic gradient, fine fraction, coefficient of erosion, deposit coefficient, dispersivity) is assessed according to experimental tests made in the laboratory.

It is observed from the results that the dispersion and deposition processes have a slight effect on soil suffusion involved in short samples. The influence of deposition kinetics may be significant for long 
samples and also for short samples subjected to severe hydraulic conditions (high gradients).

The magnitude and curve progression of the simulated evolution of the concentration and eroded mass agree well with experimental data. This model has been designed taking into account the variation of the various hydraulic and mechanical parameters. Simulation results indicate that the suffusion process is strongly linked to the mechanical and hydraulic parameters (erosion coefficient, hydraulic gradient, fines content, deposition kinetics coefficient, dispersivity). The increasing of initial fines content leads to the decrease of eroded mass fraction even if hydraulic gradient increases. The increase of sample length in direction of seepage flow reduces the eroded mass fraction. Although established by simplifying assumptions, this model allows us to quickly test the vulnerability of hydraulic structures facing the internal erosion by suffusion.

\section{ACKNOWLEDGMENTS}

This research was carried out in the framework of the formation program supported by Algerian and French governments.

\section{REFERENCES}

Ahfir, N. \& Benamar, A. \& Alem, A. \& Wang, H.Q. 2009. Influence of Internal Structure and Medium Length on Transport and Deposition of Suspended Particles: a laboratory study. Transport in Porous Media 76, 289-307

Barnichon, J. 2002. Contribution of the Bounding Surface Plasticity to the Simulation of Gallery Excavation in Plastic Clays. Engineering geology 64, 217-231

Benamar, A. \& Ahfir N. \& Wang H. \& Alem, A. 2007. Particle transport in a saturated porous medium: Pore structure effects. Compte Rendu Geoscience 339(10), 674-681

Benamar A. 2014. Effect of hydraulic load and water chemistry on soil suffusion. Proc. Int. Conf. on Scour and Erosion ICSE 7, Perth (Australia), Ed. CRC Press, pp. 197-202

Berg C.F. 2014. Permeability description by characteristic length, tortuosity, constriction and porosity. Transport in Porous Media 103, 381-400

Blume, T. \& Weisbrod, N. \& Selker, J.S. 2005. On the critical salt concentrations for particle detachment in homogeneous sand and heterogeneous Hanford sediments. Geoderma 124, 121-132

Bonelli, S. \& Marot, D. 2011: Micromechanical Modeling of Internal Erosion. European Journal of Environmental and Civil Engineering 15(8), 1207-1224

Carman P. 1937: Fluid flow through granular beds. Trans. Inst. Chem. Eng. 15, 150-166

Chapuis R.P. \& Aubertin M. 2003. On the use of the KozenyCarman equation to predict the hydraulic conductivity of soils. Can. Geotech. J. 40(3), 616-628

Einstein, A. 1906. Eine neue Bestimmung der Moleküldimensionen. Annalen der Physik 19, 289-306

Govindaraju, R.S. \& Reddi, L.N. \& Kasavaraju, S.K. 1995. A physically based model for mobilization of kaolinite particles under hydraulic gradients. Journal of Hydrology 172, $331-350$
Gravelle, A. 2011: Experimental Investigation and Modelling of Colloidal Release in Porous Media. Transport in Porous Media 88, 441-459

Khilar, K.C. \& Fogler, H.S. 1984. The existence of a critical salt concentration for particle release. Journal of Colloid and Interface Science 101, 214-224

Koponen A. \& Kataja M. \& Timonen J. 1997. Permeability and effective porosity of porous media. Physical Rev. E. 56(3), 3319

Mesticou, Z. \& Kacem, M. \& Dubujet P. 2014. Influence of Ionic Strength and Flow Rate on Silt Particle Deposition and Release in Saturated Porous Medium. Experiment and Modeling. Transport in Porous Media 103, 1-24

Nocito-Gobel, J. \& Tobiason, J.E. 1996. Effects of Ionic Strength on Colloid Deposition and Release. Colloids and Surfaces A 107, 223-231

Papamichos, E. \& Vardoulakis, I. 2005. Sand Erosion with a Porosity Diffusion law. Computers and Geotechnics 32, 4758

Roy, S.B. \& Dzombak, D.A. 1996: Colloid Release and Transport Processes in Natural and Model Porous Media. Colloids and Surfaces A 107, 245-262

Seghir, A. \& Benamar, A. \& Huaqing, W. 2014. Effects of fine particles on the suffusion of cohesionless soils. Transport in Porous Media 247, 103-233

Sterpi, D, 2003. Effects of the Erosion and Transport of Fine Particles due to Seepage Flow. International Journal of Geomechanics 3(1), 111-122

Trani L.D.O. \& Indraratna B. 2010. The use of particle size distribution by surface area method in predicting the saturated hydraulic conductivity of graded granular soils. Geotechnique 60 (12), 957-962

Vardoulakis, I. \& Stavropoulou, M., Papanastasiou, P. 1996. Hydro-mechanical Aspects of the Sand Production Problem. Transport in Porous Media 22, 225-244

Wan, C.F. \& R. Fell. 2004. Investigation of Rate of Erosion of Soils in Embankment Dams. Journal of Geotechnical and Geoenvironmental Engineering 130(4), 373-380 